\title{
THE COMPLEX BORDISM OF GROUPS WITH PERIODIC COHOMOLOGY
}

\author{
ANTHONY BAHRI, MARTIN BENDERSKY, DONALD M. DAVIS, AND \\ PETER B. GILKEY
}

\begin{abstract}
Is is proved that if $B G$ is the classifying space of a group $G$ with periodic cohomology, then the complex bordism groups $M U_{*}(B G)$ are obtained from the connective $K$-theory groups $k u_{*}(B G)$ by just tensoring up with the generators of $M U_{*}$ as a polynomial algebra over $k u_{*}$. The explicit abelian group structure is also given. The bulk of the work is the verification when $G$ is a generalized quaternionic group.
\end{abstract}

\section{Statement OF Results}

It is well known [CE] that a finite group has periodic cohomology if and only if its Sylow subgroups are all cyclic or generalized quaternionic. Another characterization $[\mathrm{Sw}]$ is that these are precisely the finite groups which can act freely on a finite simplicial homotopy sphere. In [Wo], it was shown exactly which of these (the spherical space-form groups) admit a free orthogonal action on a standard sphere.

Let $M U_{*}()$ denote (reduced) complex bordism and $b u_{*}()$ connective $K$ theory homology. It is well known [CF1] that if $B G$ denotes the classifying space of a finite group $G$, then $M U_{n}\left(B G_{+}\right)$is isomorphic to the group of bordism classes of stably almost complex $n$-manifolds with free $G$-action. Here and elsewhere $X_{+}$is the space obtained from $X$ by adjoining a disjoint basepoint. The coefficient rings are $M U_{*} \equiv M U_{*}\left(S^{0}\right)=\mathbf{Z}\left[x_{2 i}: i \geq 1\right]$ and $b u_{*} \equiv b u_{*}\left(S^{0}\right)=\mathbf{Z}\left[x_{2}\right]$, where $x_{2 i}$ is a generator of degree $2 i$ in a polynomial algebra.

Our main result proves an extension of a conjecture of Gilkey [G, BD].

Theorem 1.1. If $G$ is any finite group with periodic cohomology, then there is an isomorphism of graded abelian groups

$$
M U_{*}(B G) \approx b u_{*}(B G) \otimes \mathbf{Z}\left[x_{2 i}: i \geq 2\right] .
$$

Received by the editors May 1, 1988 .

1980 Mathematics Subject Classification (1985 Revision). Primary 57R85; Secondary 55R35, $55 \mathrm{~N} 22$.

Key words and phrases. Classifying spaces of finite groups, equivariant complex bordism, $K$ theory, groups with periodic cohomology.

The third and fourth authors were supported by National Science Foundation research grants. 
Remark. In [BD], we show that such an isomorphism does not hold for $G=$ $\mathbf{Z}_{2} \times \mathbf{Z}_{2}$.

We also obtain the explicit graded abelian group structure from 1.1. To state this, it is convenient to localize at a prime $p$. Then $M U_{(p)}$ splits as a wedge of suspensions of the Brown-Peterson spectrum $B P[\mathrm{BP}]$, so that for any space $X$

$$
M U_{*}(X)_{(p)} \approx B P_{*}(X) \otimes \mathbf{Z}_{(p)}\left[x_{2 i}: i+1 \text { is not a } p \text {-power }\right] .
$$

When working with $B P$, it is customary to denote $x_{2\left(p^{i}-1\right)}$ as $v_{i}$. Also, there is a spectrum $l$, sometimes called $B P(1)$, such that $b u_{(p)} \simeq \bigvee_{i=0}^{p-2} \Sigma^{2 i} l$ [Ad]. Then $1.2(\mathrm{a})$ below is clearly equivalent to 1.1 , where we emphasize that all isomorphisms are as graded abelian groups, i.e., no module structure is asserted. We denote by $\Sigma^{n} G$ the graded abelian group whose only nonzero component is $G$ in grading $n$.

Theorem 1.2. Let $G$ be any group with periodic cohomology, $p$ any prime, and $H$ a p-Sylow subgroup of $G$.

(a) $B P_{*}(B G) \approx l_{*}(B G) \otimes \mathbf{Z}_{(p)}\left[v_{i}: i>1\right]$;

(b) If $H=\mathbf{Z} / p^{r}$, let $k$ denote the order of the group of automorphisms of $H$ induced by inner automorphisms of $G$. The calculation of $k$ for certain groups $G$ is discussed in [CE, XII, expl. 11]. Note that $k$ is a divisor of $p-1$ and of $|G / H|$. Then

$$
l_{*}(B G) \approx \bigotimes_{i=1}^{(p-1) / k} \Sigma^{2 i k} C_{r},
$$

where

$$
C_{r}=\bigoplus_{s=0}^{r-1} \bigoplus_{l_{1}, l_{2}=0}^{p^{s}-1} \bigoplus_{j=0}^{\infty} \Sigma^{2 p^{s}-3+2(p-1)\left(l_{1}+l_{2}+p^{s} j\right)} \mathbf{Z} / p^{r-s+j} .
$$

(c) If $H=Q_{2^{m-1}}$ is a generalized quaternionic group of order $2^{m+1}$ with presentation

$$
\left\langle x, y: x^{2^{m-1}}=y^{2}, y x y^{-1}=x^{-1}\right\rangle,
$$

let

$$
\lambda=\operatorname{dim}_{\mathrm{Z}_{2}} H_{1}(B G) \in\{0,1,2\} .
$$

Then

$$
l_{*}(B G) \approx \lambda A \oplus B_{m} \oplus B_{m}^{\prime},
$$

where $\lambda A$ denotes the direct sum of $\lambda$ copies of the graded abelian group $A$, with

$$
A=\left(\Sigma^{0}+\Sigma^{2}\right) \bigoplus_{j=0}^{\infty} \Sigma^{4 j+1} \mathbf{Z} / 2^{j+1}
$$




$$
\begin{gathered}
B_{m}=\left(\Sigma^{0}+\Sigma^{2}\right)\left(\bigoplus_{j=0}^{\infty} \Sigma^{4 j+3} \mathbf{Z} / 2^{m+2 j+1}\right), \\
B_{m}^{\prime}=\left(\Sigma^{0}+\Sigma^{2}\right)\left(\bigoplus_{s=0}^{m-2} \bigoplus_{l_{1}, l_{2}=0}^{2^{s}-1} \bigoplus_{j=1}^{\infty} \Sigma^{4\left(l_{1}+l_{2}+2^{s} j\right)+3} \mathbf{Z} / 2^{m-s+j-2}\right) .
\end{gathered}
$$

Note that parts (b) and (c) contain as special cases the result when $G=H$ is $p$-primary; here $k=1$ in (b) and $\lambda=2$ in (c). We will call $B_{m} \oplus B_{m}^{\prime}$ the $B$-summand or the $B$-part.

Remark 1.3. The reader may find helpful the following table of 2-powers in summands of the $B$-part of $l_{4 n \pm 1}\left(B Q_{2^{m-1}}\right), n \leq 7 . B_{m}$ corresponds to the

\begin{tabular}{|c|c|c|c|c|c|c|c|}
\hline$n$ & \multicolumn{7}{|c|}{ exponents of 2 in summands } \\
\hline 1 & $m+1$ & & & & & & \\
\hline 2 & $m+3$ & $m-1$ & & & & & \\
\hline 3 & $m+5$ & $m$ & $m-2$ & & & & \\
\hline 4 & $m+7$ & $m+1$ & $m-2$ & $m-2$ & & & \\
\hline 5 & $m+9$ & $m+2$ & $m-1$ & $m-2$ & $m-3$ & & \\
\hline 6 & $m+11$ & $m+3$ & $m-1$ & $m-1$ & $m-3$ & $m-3$ & \\
\hline 7 & $m+13$ & $m+4$ & $m$ & $m-1$ & $m-3$ & $m-3$ & $m-3$ \\
\hline
\end{tabular}
first column of the table, while the remaining columns correspond to $B_{m}^{\prime}$.

$\S \S 2$ and 3 are devoted to the proofs of Theorems 1.1 and 1.2. In $\S 4$ we discuss an algebraic conjecture arising from these calculations. In $\S 5$ we give a counterexample to a conjecture of [BD], which speculated that 1.1 might be true if $B G$ is replaced by any space $X$ such that hom $\operatorname{dim}_{M U_{*}}\left(M U_{*} X\right) \leq 1$.

\section{SKETCH OF PROOF}

In [BD], we cited the calculation of $l_{*}\left(B Z / p^{r}\right)$ in [Ha] and proved 1.2(a) when $G=\mathbf{Z} / p^{r}$. In $\S 3$, we carry out a similar program when $G$ is a generalized quaternionic group. Here the result for $l_{*}\left(B Q_{2^{m-1}}\right)$ follows easily from the calculation of $\widetilde{K}\left(S^{4 n+3} / Q_{2^{m-1}}\right)$ in [FS], but showing that $B P_{*}\left(B Q_{2^{m-1}}\right)$ is as claimed in 1.2(a) constitutes the bulk of this paper. 
This then gives 1.2(a) for all Sylow subgroups of a group $G$ with periodic cohomology, and 1.2(a) for $G$ then follows easily from

Theorem $2.1[C]$. For any group $G$ with p-Sylow subgroup $H$, there is a spectrum $W$ such that the suspension spectrum of $\mathrm{BH}_{+}$splits into a wedge of p-local spectra

$$
B H_{+} \simeq B G_{+(p)} \vee W
$$

where the first is the p-localization of the suspension spectrum of $B G_{+}$.

To deduce 1.2(a) and hence 1.1, we use the spectral sequence (SS) introduced in [Jo]

$$
l_{*}(X) \otimes \mathbf{Z}_{(p)}\left[v_{i}: i \geq 2\right] \Rightarrow B P_{*}(X) .
$$

Since all spectra considered here have $l$-homology zero in even dimensions, this SS clearly collapses (all differentials zero) for dimensional reasons; however, as discussed in [BD], the crux is the extensions in this SS. Our proof of 1.2(a) for cyclic and generalized quaternionic groups $H$ shows that for $X=B H$ the SS (2.2) has no nontrivial extensions, i.e.,

$$
B P_{n}(X) \approx \bigoplus_{j} l_{j}(X) \otimes \mathbf{Z}_{(p)}\left[v_{i}: i \geq 2\right]_{n-j} .
$$

Theorem 2.1 shows that the SS $(2.2)$ for $X=B G_{(p)}$ is a direct summand in the SS for $X=B H$, and hence it too has no nontrivial extensions, implying $1.2(\mathrm{a})$.

The explicit determination of $l_{*}(B G)$ when $G$ has periodic cohomology and is not $p$-primary utilizes the well-known calculation of $H_{*}(B G)$.

Theorem 2.3 [Sw]. If $G$ has periodic cohomology and $H$ is a p-Sylow subgroup, then

(i) if $H$ is cyclic and $k$ is as in 1.2(b), then

$$
H_{i}\left(B G ; \mathbf{Z}_{(p)}\right) \approx \begin{cases}H & \text { if } i+1 \equiv 0 \quad \bmod 2 k \\ 0 & \text { otherwise }\end{cases}
$$

(ii) if $p=2$ and $H=Q_{2^{m-1}}$ is a generalized quaternionic group, then $H_{1}\left(B G ; \mathbf{Z}_{(2)}\right)$ is a direct summand of $H_{1}\left(B H ; \mathbf{Z}_{(2)}\right) \approx \mathbf{Z}_{2} \oplus \mathbf{Z}_{2}$, and

$$
\left.H_{i}(B G ; \mathbf{Z})_{(2)}\right) \approx \begin{cases}\mathbf{Z} / 2^{m+1} & \text { if } i \equiv 3 \bmod 4, \\ H_{1}\left(B G ; \mathbf{Z}_{(2)}\right) & \text { if } i \equiv 1 \bmod 4, \\ 0 & \text { if } i \text { even and positive. }\end{cases}
$$

Let $\operatorname{AHSS}(X)$ denote the Atiyah-Hirzebruch SS

$$
H_{*}\left(X ; \mathbf{Z}_{(p)}\right) \otimes \mathbf{Z}_{(p)}\left[v_{1}\right] \Rightarrow l_{*}(X) .
$$

Suppose $G$ is a group whose $p$-Sylow subgroup $H$ is cyclic. The $\operatorname{AHSS}(B H)$ collapses but has nontrivial extensions, and, since $\left|v_{1}\right|=2(p-1)$, it splits formally as a sum of $p-1$ SS's, one for total degree each odd residue 
mod 2(p-1). By 2.1 and 2.3(i), $\operatorname{AHSS}(B G)$ is just the sum of the summands in total degree one less than a multiple of $2 k$, establishing $1.2(\mathrm{~b})$.

If $G$ is a group whose 2-Sylow subgroup $H$ is a generalized quaternionic group, the $\operatorname{AHSS}(B H)$ again collapses and has nontrivial extensions, which have an interesting pattern described at the end of $\S 3$. It splits as in 1.2(c) into two $A$-summands and a $B$-summand. In fact, it was shown in [MP] that this is induced by a splitting of the suspension spectrum of $B H$. By 2.3(ii) and 2.1, $\operatorname{AHSS}(B G)$ will be the sum of the $B$-summand and the appropriate number of $A$-summands, establishing $1.2(\mathrm{c})$.

\section{PROOF FOR GENERALIZED QUATERNIONIC GROUPS}

Let $Q=Q_{2^{m-1}}$ be the generalized quaternionic group of order $2^{m+1}$. We cull from [FS, 1.6, 1.7, 5.10] the following result, where $S^{4 n+3} / Q$ denotes the quotient manifold, which is the $(4 n+3)$-skeleton of $B Q$.

Theorem 3.1 [FS]. There are canonical $U(1)$-bundles $a_{\varepsilon}(\varepsilon=0,1)$ and a $U(2)$ bundle $b$ over $S^{4 n+3} / Q$ whose cohomology Chern classes satisfy

$$
c_{1}\left(a_{\varepsilon}\right) \neq 0 \quad \text { and } \quad c_{1}(b)=0 .
$$

Let $\mathscr{K}^{n}$ denote the subring of $\widetilde{K}\left(S^{4 n+3} / Q\right)$ generated by $\beta=\{b-2\}$. Let $N=\min \left\{n, 2^{m-1}\right\}$ throughout this section.

(i) $\mathscr{K}^{n} \approx \beta \cdot \mathbf{Z}[\beta] /\left(\beta^{n+1}, P(\beta)\right)$, where $P(\beta)$ is a polynomial which does not depend on $n$ and has lowest term $2^{m+1} \beta$.

(ii) For $i=1, \ldots, N$, there are elements $\delta_{i}^{(n)}=\sum_{k=1}^{i} d_{k} \beta^{k}$ with $d_{i}$ odd and $d_{k}$ even if $k<i$, which generate summands in a direct sum decomposition of $\mathscr{K}^{n}$ such that the order of the $\delta_{i}^{(n)}$-summand equals that of the summand in 1.2(c) determined as follows:

$$
\begin{gathered}
\text { if } i=1, \text { then } j=n-1 \text { in } B_{m} ; \quad \text { if } 2^{s}<i \leq 2^{s+1} \text {, then in } B_{m}^{\prime} \\
l_{1}=i-2^{s}-1, j=\left[\left(n-1-l_{1}\right) / 2^{s}\right], \text { and } l_{2}=n-1-l_{1}-2^{s} j .
\end{gathered}
$$

(iii) There is a certain polynomial $Q(\beta)$ such that if $\alpha_{1}^{(n)}=\left\{\alpha_{1}-1\right\}-Q(\beta)$ and $\alpha_{0}^{(n)}=\left\{a_{0}-1\right\}$, then $\widetilde{K}\left(S^{4 n+3} / Q\right) \approx \mathscr{K}^{n} \oplus\left\langle\alpha_{0}^{(n)}\right\rangle \oplus\left\langle\alpha_{1}^{(n)}\right\rangle$, where the latter two summands have order $2^{n+1}$.

That $l_{*}(B Q)\left(=b u_{*}(B Q)\right.$ since $\left.p=2\right)$ is as claimed in 1.2(c) follows from 3.1 and the isomorphisms

$$
\begin{gathered}
b u_{4 n+1}(B Q) \approx b u_{4 n+1}\left(S^{4 n+3} / Q\right) \approx b u^{2}\left(S^{4 n+3} / Q\right) \approx \widetilde{K}\left(S^{4 n+3} / Q\right), \\
b u_{4 n-1}(B Q) \approx b u_{4 n-1}\left(S^{4 n+3} / Q\right) \approx b u^{4}\left(S^{4 n+3} / Q\right) \\
\approx \operatorname{ker}\left(\widetilde{K}\left(S^{4 n+3} / Q\right) \rightarrow \widetilde{K}\left(S^{3} / Q\right)\right) .
\end{gathered}
$$


By the commutative diagram

$$
\begin{array}{ccc}
\widetilde{K}\left(S^{4 n+3} / Q\right) & \stackrel{i^{*}}{\longrightarrow} & \widetilde{K}\left(S^{3} / Q\right) \\
\downarrow c_{1} & \approx \downarrow c_{1} \\
H^{2}\left(S^{4 n+3} / Q\right) \stackrel{i^{*}}{\longrightarrow} H^{2}\left(S^{3} / Q\right)=\mathbf{Z}_{2} \oplus \mathbf{Z}_{2}
\end{array}
$$

and (3.2), the kernel in the second part of (3.3) is $\mathscr{K}^{n} \oplus\left\langle 2 \alpha_{0}^{(n)}\right\rangle \oplus\left\langle 2 \alpha_{1}^{(n)}\right\rangle$. Here the latter two summands have order $2^{n}$, giving the second $\left(\Sigma^{2}\right) A$-summands in $1.2(\mathrm{c})$ with $j=n-1$.

It remains to prove 1.2 (a) when $G=Q$. The idea is to use the Conner-Floyd classes of the $K$-theory generators of 3.1. Some work is required to show that these can be chosen to have the same order as the $K$-theory classes and that they span.

For $n \geq 0$ and $\varepsilon=0,1$, let $A_{\varepsilon}^{(n)}=D\left(\operatorname{cf}_{1}\left(\alpha_{\varepsilon}^{(n)}\right)\right) \in B P_{4 n+1}(B Q)$ denote the image of the first Conner-Floyd class under the isomorphism

$$
D: B P^{2}\left(S^{4 n+3} / Q\right) \approx B P_{4 n+1}\left(S^{4 n+3} / Q\right) \approx B P_{4 n+1}(B Q) .
$$

Since $\mathrm{cf}_{1}()$ is linear,

$$
2^{n+1} A_{\varepsilon}^{(n)}=0 .
$$

$\mathrm{cf}_{2}()$ is not linear, but $\mathrm{cf}_{2}()-\frac{1}{2} \mathrm{cf}_{1}()^{2}$ is, when it is defined. The following result, proved later in this section, will be used to give an analog of this linear class.

Proposition 3.5. If $2^{u}=\operatorname{order}\left(\delta_{i}^{(n)}\right)$, then $2^{u-1}\left(\operatorname{cf}_{1}\left(\delta_{i}^{(n)}\right)\right)^{2}$ is divisible by $2^{u}$ in $B P^{4}\left(S^{4 n+3} / Q\right)$.

Let

$$
\operatorname{cf}_{2}^{\prime}\left(\delta_{i}^{(n)}\right)=\operatorname{cf}_{2}\left(\delta_{i}^{(n)}\right)-\frac{2^{u-1}\left(\mathrm{cf}_{1}\left(\delta_{i}^{(n)}\right)\right)^{2}}{2^{u}} \in B P^{4}\left(S^{4 n+3} / Q\right) .
$$

This division by $2^{u}$ does not yield a well-defined element; it can be varied by any element annihilated by $2^{u}$. In the proof of 3.5 , an explicit choice will be made. Since

$$
0=\operatorname{cf}_{2}(0)=\operatorname{cf}_{2}\left(2^{u} \delta_{i}^{(n)}\right)=2^{u} \operatorname{cf}_{2}\left(\delta_{i}^{(n)}\right)+\left(\begin{array}{c}
2^{u} \\
2
\end{array}\right)\left(\mathrm{cf}_{1}\left(\delta_{i}^{(n)}\right)\right)^{2}
$$

and

$$
\left(\begin{array}{c}
2^{u} \\
2
\end{array}\right) \equiv 2^{u-1} \quad \bmod 2^{u} \quad \text { and } \quad 2^{u} \operatorname{cf}_{1}\left(\delta_{i}^{(n)}\right)=\operatorname{cf}_{1}\left(2^{u} \delta_{i}^{(n)}\right)=0 \text {, }
$$

we deduce $2^{u} \operatorname{cf}_{2}^{\prime}\left(\delta_{i}^{(n)}\right)=0$. Let $B_{i}^{(n)}=D\left(\operatorname{cf}_{2}^{\prime}\left(\delta_{i}^{(n)}\right)\right) \in B P_{4 n-1}(B Q)$, where

$$
D: B P^{4}\left(S^{4 n+3} / Q\right) \stackrel{\approx}{\longrightarrow} B P_{4 n-1}(B Q)
$$

is similar to the previous $D$. Then

$$
2^{u} B_{i}^{(n)}=0
$$


Later in this section we will prove

Proposition 3.7. As a $\mathbf{Z}_{(2)}\left[v_{2}, v_{3}, \ldots\right]$-module, $B P_{*}(B Q)$ is spanned by $\left\{v_{1}^{e} A_{\varepsilon}^{(n)}: 0 \leq e \leq 1,0 \leq \varepsilon \leq 1, n \geq 0\right\} \cup\left\{v_{1}^{e} B_{i}^{(n)}: 0 \leq e \leq 1, n \geq 1,1 \leq i \leq N\right\}$. Remark. The $v_{1}^{e}$ in 3.7 correspond to $\Sigma^{0}+\Sigma^{2}$ in $1.2(\mathrm{c})$. Higher powers of $v_{1}$ are subsumed in $A$ 's and $B$ 's with larger superscripts.

1.2 (a) for $G=Q$ is now immediate. Let $\mathscr{G}$ denote the graded abelian group with direct summands corresponding to all pairs $\left(v^{E}, x\right)$, where $v^{E}$ is a monomial in $v_{2}, v_{3}, \ldots$, and $x$ is in the spanning set of 3.7, with order of summand equal to the 2-power which 3.4 or 3.6 says annihilates the relevant $x$. Define a homomorphism $\mathscr{G} \rightarrow B P_{*}(B Q)$ by sending abstract generators to the appropriate element $v^{E} x$. This is well defined by 3.4 and 3.6 , it is surjective by 3.7 , and since the graded abelian groups $\mathscr{G}$ and $B P_{*}(B Q)$ have the same orders, namely that of $l_{*}(B Q) \otimes \mathbf{Z}_{(2)}\left[v_{2}, \ldots\right]$, it is an isomorphism. [That $B P_{*}(B Q)$ has this order follows from the collapsing of the SS (2.2).]

Thus it remains to prove 3.5 and 3.7. The following result, which we extract from [Mes], will be useful for each. We provide a simplification of the proof of [Mes].

Lemma 3.8. Let $\mathscr{B}^{n}$ denote the sub- $B P^{*}$-algebra of $B P^{*}\left(S^{4 n+3} / Q\right)$ generated by $Y=\mathrm{cf}_{2}(\beta)$. Then

(i) $\mathscr{B}^{n} \approx Y \cdot B P^{*}[Y] /\left(Y^{n+1}, P_{1}(Y)\right)$, where $P_{1}(Y)$ is a polynomial which does not depend on $n$ and has lowest term $2^{m+1} Y$;

(ii) $\operatorname{cf}_{j}\left(\beta^{k}\right) \in \mathscr{B}^{n}$ for any $k \geq 1$ and $j \geq 1$.

Proof. Let $i: S^{4 n+3} / Q \rightarrow S^{4 n+3} / S p(1)=H P^{n}$ denote the map induced by the group inclusion $Q \rightarrow S p(1)$. The canonical $U(2)$-bundle $\theta$ over $H P^{n}$ satisfies $i^{*}\{\theta-2\}=\beta$. Then $\mathscr{B}^{n}$ is the image of $B P^{*}\left(H P^{n}\right) \stackrel{i^{*}}{\longrightarrow} B P^{*}\left(S^{4 n+3} / Q\right)$, and (ii) is clear since $\operatorname{cf}_{j}\left(\beta^{k}\right)=\operatorname{cf}_{j}\left(i^{*} \theta^{k}\right)=i^{*}\left(\operatorname{cf}_{j}\left(\theta^{k}\right)\right)$.

To determine the polynomial $P_{1}$, let $b$ be the bundle of 3.1. Then $b^{k}$ is classified by

$$
S^{4 n+3} / Q \stackrel{\Delta}{\longrightarrow}\left(S^{4 n+3} / Q\right)^{k} \stackrel{g}{\longrightarrow} B U(2)^{k} \stackrel{m}{\longrightarrow} B U\left(2^{k}\right),
$$

where $g$ is the Cartesian product of $k$ maps classifying $b$. For $1 \leq i \leq k$, let $b_{i}$ denote the pullback of $b$ under the projection map from $\left(S^{4 n+3} / Q\right)^{k}$ to its $i$ th factor, and let $\left(S^{4 n+3} / Q\right)_{i}$ denote the subspace of $\left(S^{4 n+3} / Q\right)^{k}$ consisting of tuples whose $j$ th component is the basepoint whenever $j \neq i$. Then the restriction of $m \circ g$ to $\left(S^{4 n+3} / Q\right)_{i}$ is $2^{k-1} b_{i}$, and hence $b^{\times k}-\bigoplus_{i=1}^{k} 2^{k-1} b_{i}$ is in the image of

$$
K\left(\left(S^{4 n+3} / Q\right)^{k} / \bigcup_{i}\left(S^{4 n+3} / Q\right)_{i}\right) \rightarrow K\left(\left(S^{4 n+3} / Q\right)^{k}\right),
$$


from which follows the first " $\equiv$ " in

$$
\mathrm{cf}_{2}\left(b^{k}\right) \equiv k \cdot \mathrm{cf}_{2}\left(2^{k-1} b\right) \equiv k 2^{k-1} \mathrm{cf}_{2}(b) \quad \bmod \left(Y^{2}\right) .
$$

Thus, $\bmod \left(Y^{2}\right)$,

$$
\begin{aligned}
\operatorname{cf}_{2}\left(\beta^{k}\right) & =\operatorname{cf}_{2}\left(\sum_{j=0}^{k}(-1)^{j}\left(\begin{array}{c}
k \\
j
\end{array}\right) 2^{j} b^{k-j}\right) \equiv \sum_{j=0}^{k-1}(-1)^{j}\left(\begin{array}{c}
k \\
j
\end{array}\right) 2^{j}(k-j) 2^{k-j-1} Y \\
& =2^{k-1} Y \sum_{j=0}^{k-1}(-1)^{j} k\left(\begin{array}{c}
k-1 \\
j
\end{array}\right)= \begin{cases}Y, & k=1, \\
0, & k>1 .\end{cases}
\end{aligned}
$$

Now if $P=\sum_{k \geq 1} a_{k} \beta^{k}$ is as in 3.1(i) $\left(a_{1}=2^{m+1}\right)$, then

$$
\begin{aligned}
0= & \operatorname{cf}_{2}\left(\sum a_{k} \beta^{k}\right)=\sum a_{k} \mathrm{cf}_{2}\left(\beta^{k}\right)+\sum a_{k \neq j} a_{j} \operatorname{cf}_{1}\left(\beta^{j}\right) \mathrm{cf}_{1}\left(\beta^{k}\right) \\
& +\sum\left(\begin{array}{c}
a_{k} \\
2
\end{array}\right) \mathrm{cf}_{1}\left(\beta^{k}\right)^{2},
\end{aligned}
$$

which can be expressed as a polynomial $P_{1}(Y)$. By (3.9), the only $Y^{1}$-part is $a_{1} \operatorname{cf}_{2}\left(\beta^{1}\right)=2^{m+1} Y$.

Thus there is a well-defined epimorphism

$$
Y \cdot B P_{*}[Y] /\left(P_{1}(Y), Y^{n+1}\right) \rightarrow \mathscr{B}^{n},
$$

which is bijective because both have the order of $Y \cdot B P_{*}[Y] /\left(2^{m+1}, Y^{n+1}\right)$. The order of $\mathscr{B}^{n}$ is calculated by the AHSS for the $B$-summand of the [MP] splitting discussed at the end of $\S 2$.

Proof of 3.5. Let $\rho: \mathscr{K}^{n} \rightarrow \mathscr{K}^{i-1}$ and $\rho_{B}: \mathscr{B}^{n} \rightarrow \mathscr{B}^{i-1}$ denote restriction. Then

$$
\rho_{B}\left(\mathrm{cf}_{1}\left(\beta^{i}\right)\right)=\operatorname{cf}_{1}\left(\rho\left(\beta^{i}\right)\right)=\operatorname{cf}_{1}(0)=0 .
$$

Thus by $3.8(\mathrm{i}), \mathrm{cf}_{1}\left(\beta^{i}\right)$ is a multiple of $Y^{i}$, say $Y^{i} q(Y)$. Now we break into two cases.

Case 1. $2 i>n$. Using linearity of $\mathrm{cf}_{1}(\mathrm{)})$ and 3.1 (ii) at the first step, and $2 i>n$ at the last, we have

$$
\left(\mathrm{cf}_{1}\left(\delta_{i}^{(n)}\right)\right)^{2} \equiv\left(\mathrm{cf}_{1}\left(\beta^{i}\right)\right)^{2}=Y^{2 i} q(Y)^{2}=0 \bmod 4 .
$$

Thus $2^{u-1}\left(\operatorname{cf}_{1}\left(\delta_{i}^{(n)}\right)\right)^{2}$ is divisible by $2^{u+1}$ for any $u$.

Case 2. $2 i \leq n$. Since $i \leq n-i$, there is an element $\delta_{i}^{(n-i)}$, and it satisfies $2^{u-1} \delta_{i}^{(n-i)}=0$. [From 3.1(ii), decreasing $n$ by $i$ decreases $j$ by 1 or 2 , and hence decreases the order.] By the explicit formula for $\delta_{i}$ in [FS, 1.6], there is an element $\gamma$ so that $\rho^{\prime}: \mathscr{K}^{n} \rightarrow \mathscr{K}^{n-i}$ sends $\delta_{i}^{(n)}+2 \gamma$ to $\delta_{i}^{(n-i)}$. [If $i=2^{s}$, then

$$
2 \gamma=\sum_{t=1}^{s}\left(2^{\left(2^{t}-1\right)\left(\left[\frac{n-1}{2^{s-1}}\right]+1\right)}-2^{\left(2^{t}-1\right)\left(\left[\frac{n}{2^{s-1}}\right]+1\right)}\right) \beta(s-t),
$$


which is, in fact, a multiple of 8 . Here $\beta(i)$ as in [FS, p. 508] satisfies $\beta(0)=$ $\beta, \beta(i+1)=\beta(i)^{2}+4 \beta(i)$. If $i=2^{s}+d$ with $1 \leq d<2^{s}$, the least 2-divisible term in $2 \gamma$ is $2^{\left[\frac{n-2 i}{2^{s}}+1\right.} \beta^{d} \beta(s)$.]

The restriction $\rho_{B}^{\prime}: \mathscr{B}^{n} \rightarrow \mathscr{B}^{n-i}$ sends $2^{u-1} \operatorname{cf}_{1}\left(\delta_{i}^{(n)}+2 \gamma\right)$ to $2^{u-1} \operatorname{cf}_{1}\left(\delta_{i}^{(n-i)}\right)$ $=0$. Hence $2^{u-1} \mathrm{cf}_{1}\left(\delta_{i}^{(n)}+2 \gamma\right)$ is divisible by $Y^{n-i+1}$; call it $Y^{n-i+1} r(Y)$. Then

$$
\begin{aligned}
2^{u-1} \operatorname{cf}_{1}\left(\delta_{i}^{(n)}\right)^{2} & =2^{u-1} \operatorname{cf}_{1}\left(\delta_{i}^{(n)}\right) \operatorname{cf}_{1}\left(\beta^{i}\right) \\
& =\left(Y^{n-i+1} r(Y)-2^{u} \operatorname{cf}_{1}(\gamma)\right) Y^{i} q(Y) \\
& =-2^{u} \operatorname{cf}_{1}(\gamma) Y^{i} q(Y),
\end{aligned}
$$

where the first equality used $2^{u} \mathrm{cf}_{1}\left(\delta_{i}^{(n)}\right)=0$.

Let $\mu: B P_{*}(X) \rightarrow b u_{*}(X)$ denote the Conner-Floyd homomorphism [CF2]. We will prove

Proposition 3.10. bu $u_{*}(B Q)$ is spanned by $\left\{v_{1}^{e} \mu\left(A_{\varepsilon}^{(n)}\right)\right\} \cup\left\{v_{1}^{e} \mu\left(B_{i}^{(n)}\right)\right\}$, with same indices as in 3.7.

Then the collapsing of the Johnson SS (2.2) for $B Q$ and a standard filtration argument imply 3.7 , and hence 1.2 .

In proving 3.10 , we will have to be careful about the relationship between $K(X)$ and $b u_{*}(X)$. Before introducing these issues, we give a lemma in $K$ theory. Throughout the remainder of the paper, let $c_{i}()$ denote the $K$-theory Chern classes.

Lemma 3.11. In $\widetilde{K}\left(S^{4 n+3} / Q\right), c_{2}\left(\beta^{i}\right) \equiv \beta^{i} \bmod 2$ for any $i \leq n$.

Proof. We use the standard facts that for $x \in \widetilde{K}(X) \psi^{2}(x)=x^{2}-2 \lambda^{2}(x)[\mathrm{Hu}$, p. 161] and $c_{2}(x)=x+\lambda^{2}(x)$ [At, p. 122]. Also, $\lambda^{1}=\mathrm{id}, \lambda^{2}(-2)=3$, $\left[0=\lambda^{2}(2+(-2))=\lambda^{2}(2)+2 \cdot(-2)+\lambda^{2}(-2)\right]$, and, since $b$ is an $S U(2)$-bundle, $\lambda^{2}(b)=1$. Hence

$$
\lambda^{2}(\beta)=\lambda^{2}(b)+\lambda^{1}(b) \lambda^{1}(-2)+\lambda^{2}(-2)=4-2 b=-2 \beta .
$$

Thus $\psi^{2}(\beta)=\beta^{2}+4 \beta$, and, using multiplicativity of $\psi^{2}$, we get

$$
\begin{aligned}
2 c_{2}\left(\beta^{i}\right) & =2 \beta^{i}+\left(\beta^{i}\right)^{2}-\psi^{2}\left(\beta^{i}\right)=2 \beta^{i}+\beta^{2 i}-\left(\beta^{2}+4 \beta\right)^{i} \\
& =\left(2-4^{i}\right) \beta^{i}-\sum_{j=1}^{i-1} 4^{j}\left(\begin{array}{l}
i \\
j
\end{array}\right) \beta^{2 i-j} .
\end{aligned}
$$

We can divide by 2 , introducing as indeterminacy $\operatorname{ker}(\cdot 2)$. Since $2^{m} \beta^{j} \neq 0$ for $1 \leq j \leq n$ by [KS, 1.4] and [FS, 5.10], this indeterminacy involves only large 2-powers as coefficients of $\beta^{j}$ 's.

Corollary 3.12. If $u$ is as in 3.5, then $2^{u-1}\left(c_{1}\left(\delta_{i}^{(n)}\right)\right)^{2}$ is divisible by $2^{u}$ in $\widetilde{K}\left(S^{4 n+3} / Q\right)$. If $c_{2}^{\prime}\left(\delta_{i}^{(n)}\right)$ is defined as

$$
c_{2}\left(\delta_{i}^{(n)}\right)-2^{u-1}\left(c_{1}\left(\delta_{i}^{(n)}\right)\right)^{2} / 2^{u},
$$

then $c_{2}^{\prime}\left(\delta_{i}^{(n)}\right) \equiv \beta^{i} \bmod \left(2, \beta^{i+1}\right)$. 
Proof. The mod 2 value of $c_{2}\left(\delta_{i}^{(n)}\right)$ depends on the $\bmod 4$ value of $\delta_{i}^{(n)}$. From [FS, 1.6]

$$
\delta_{i}^{(n)} \equiv \pm \beta^{i}+2 \sum k_{j} \beta^{j} \bmod 4
$$

with $k_{j}$ an integer which is even unless $i=2^{s}+d, 1 \leq d<2^{s}$, and $j \geq$ $2^{s-1}+d$. [It is congruent to $\beta^{d+1} \prod_{t=0}^{s-1}\left(2+\beta^{2^{t}}\right)+2^{p} \beta^{i}$, for some $p \geq 1$.] Since those $j$ with $k_{j}$ odd satisfy $2 j>i$, and $c_{1}=-$ id on $\widetilde{K}()$, we have

$$
c_{2}\left(\delta_{i}^{(n)}\right) \equiv c_{2}\left(\beta^{i}\right)+\sum_{k_{j} \text { odd }}\left(c_{1}\left(\beta^{j}\right)\right)^{2} \equiv \beta^{i} \bmod \left(2, \beta^{i+1}\right),
$$

using Lemma 3.11.

By the method of proof for Proposition 3.5, working with $c_{1}$ rather than $\mathrm{cf}_{1}$, $2^{u-1}\left(c_{1}\left(\delta_{i}^{(n)}\right)\right)^{2} / 2^{u}$ exists and is in $\left(2, \beta^{i+1}\right)$. [If $2 i>n$, then

$$
2^{u-1} c_{1}\left(\delta_{i}^{(n)}\right)^{2}=2^{u-1}\left(\beta^{n-i+1} r^{\prime}(\beta)-2 \gamma\right) \beta^{i}
$$

with $\gamma$ divisible by $\beta$.]

Proof of 3.10. We will use the commutative diagram

$$
\begin{array}{ccc}
b u^{4}(X) & \approx & \operatorname{ker}\left(\widetilde{K}(X) \rightarrow \widetilde{K}\left(X^{(3)}\right)\right) \\
v_{1} \downarrow & & \downarrow \\
b u^{2}(X) & \approx & \widetilde{K}(X),
\end{array}
$$

which just reflects the role of $v_{1}$ as Bott periodicity. We will identify elements in $\widetilde{K}(X)$ with the corresponding elements of $b u^{2}(X)$. We will distinguish duality isomorphisms by

$$
\begin{gathered}
D_{2}: b u^{2}\left(S^{4 n+3} / Q\right) \rightarrow b u_{4 n+1}\left(S^{4 n+3} / Q\right) \text { and } \\
D_{4}: b u^{4}\left(S^{4 n+3} / Q\right) \rightarrow b u_{4 n-1}\left(S^{4 n+3} / Q\right) .
\end{gathered}
$$

From 3.1 and 3.3, $b u_{*}(B Q)$ is spanned by $S_{1} \cup S_{1}^{\prime} \cup S_{2} \cup S_{2}^{\prime}$, where

$$
\begin{aligned}
& S_{1}=\left\{i_{*}\left(D_{2}\left(\alpha_{\varepsilon}^{(n)}\right)\right) \in b u_{4 n+1}(B Q): 0 \leq \varepsilon \leq 1, n \geq 0\right\}, \\
& S_{1}^{\prime}=\left\{i_{*}\left(D_{4}\left(v_{1}^{-1}\left(2 \alpha_{\varepsilon}^{(n)}\right)\right)\right) \in b u_{4 n-1}(B Q): 0 \leq \varepsilon \leq 1, n \geq 1\right\}, \\
& S_{2}=\left\{i_{*}\left(D_{2}\left(\theta_{i}\right)\right) \in b u_{4 n+1}(B Q): n \geq 1,1 \leq i \leq N\right\}, \\
& S_{2}^{\prime}=\left\{i_{*}\left(D_{4}\left(v_{1}^{-1} \theta_{i}\right)\right) \in b u_{4 n-1}(B Q): n \geq 1,1 \leq i \leq N\right\} .
\end{aligned}
$$

Here $i_{*}$ is the homomorphism in $b u_{*}()$ induced by the inclusion $S^{4 n+3} / Q \rightarrow$ $B Q$, and $\theta_{i}$ is any element of $\widetilde{K}\left(S^{4 n+3} / Q\right)$ such that $\theta_{i} \equiv \beta^{i} \bmod \left(2, \beta^{i+1}\right)$. [That any such set of elements spans $\widetilde{K}\left(S^{4 n+3} / Q\right)$ follows by an easy filtration argument from the fact that the $\delta_{i}^{(n)}$ are of this form.]

The elements $c_{2}^{\prime}\left(\delta_{i}^{(n)}\right)$ of 3.12 satisfy the hypotheses required of $\theta_{i}$ above. Since $\mu \circ \mathrm{cf}_{2}=c_{2}$, it follows from the definitions that

$$
i_{*}\left(D_{4}\left(v_{1}^{-1} c_{2}^{\prime}\left(\delta_{i}^{(n)}\right)\right)\right)=\mu\left(B_{i}^{(n)}\right) \quad \text { and } i_{*}\left(D_{2}\left(c_{2}^{\prime}\left(\delta_{i}^{(n)}\right)\right)\right)=v_{1} \mu\left(B_{i}^{(n)}\right),
$$

so that the $\left\{v_{1}^{e} \mu\left(B_{i}^{(n)}\right)\right\}$ of our 3.10 works as $S_{2} \cup S_{2}^{\prime}$ above. 
The elements $\mu\left(A_{\varepsilon}^{(n)}\right)$ in our 3.10 are up to sign the elements of $S_{1}$, since $\mu\left(\mathrm{cf}_{1}(\alpha)\right)=-\alpha$.

To show that the element $v_{1} \mu\left(A_{\varepsilon}^{(n-1)}\right)$ in our 3.10 equals $-i_{*} D_{4}\left(v_{1}^{-1}\left(2 \alpha_{\varepsilon}^{(n)}\right)\right)$ $\in S_{1}^{\prime}$, we must show

$$
v_{1} j_{*} D_{2}\left(\alpha_{\varepsilon}^{(n-1)}\right)=D_{4}\left(v_{1}^{-1}\left(2 \alpha_{\varepsilon}^{(n)}\right)\right) \quad \text { in } b u_{4 n-1}\left(S^{4 n+3} / Q\right) .
$$

Here we have used that $\mu$ commutes with $D$ and that $\mu \circ \mathrm{cf}_{1}=-1$. The homomorphism $j_{*}$ is induced by the inclusion $S^{4 n-1} / Q \stackrel{j}{\longrightarrow} S^{4 n+3} / Q$. After commuting $D$ with $v_{1}$, this reduces to the fact that the composite

$$
\begin{aligned}
b u^{2}\left(S^{4 n-1} / Q\right) & \stackrel{D_{2}}{\longrightarrow} b u_{4 n-3}\left(S^{4 n-1} / Q\right) \stackrel{j_{*}}{\longrightarrow} b u_{4 n-3}\left(S^{4 n+3} / Q\right) \\
& \stackrel{v_{1}^{2}}{\longrightarrow} b u_{4 n+1}\left(S^{4 n+3} / Q\right) \stackrel{D_{2}^{-1}}{\longrightarrow} b u^{2}\left(S^{4 n+3} / Q\right)
\end{aligned}
$$

sends $\alpha_{\varepsilon}^{(n-1)}$ to $2 \alpha_{\varepsilon}^{(n)}$, up to odd multiples. This follows from the nontrivial extension in $\operatorname{AHSS}\left(S^{4 n+3} / Q\right)$ from $D_{2}\left(\alpha_{\varepsilon}^{(n)}\right) \otimes 1$ to $j_{*}\left(D_{2}\left(\alpha_{\varepsilon}^{(n-1)}\right)\right) \otimes v_{1}^{2}$. This SS will be discussed in more detail later in this section. This completes the formal part of the proof.

We end this section by addressing some comments about SS's made earlier. First, the triviality of extensions in the Johnson SS (2.2) for $X=B Q$ :

Let $P_{j}$ denote the summand of $\mathbf{Z}_{(2)}\left[v_{2} \ldots\right]$ in grading $j$. The $\mathrm{SS}$ says there is a filtration

$$
0=\mathscr{F}_{n} \subset \cdots \subset \mathscr{F}_{1} \subset \mathscr{F}_{0}=B P_{n}(B Q)
$$

with $\mathscr{F}_{i} / \mathscr{F}_{i+1} \approx b u_{n-i}(B Q) \otimes P_{i}$. A splitting map $s$ for the short exact sequence

$$
0 \rightarrow \mathscr{F}_{i+1} \rightarrow \mathscr{F}_{i} \stackrel{s}{\leftrightarrows} \mathscr{F}_{i} / \mathscr{F}_{i+1} \rightarrow 0
$$

is given by $s\left(\mu(x) \otimes v^{E}\right)=v^{E} x$, where $x$ and $v^{E}$ are as in the proof of 1.2(a) which follows 3.7. Here we are using 3.10.

The $\operatorname{AHSS}(B Q),(2.4)$, is much more interesting. By the [MP] splitting of $B Q$, the SS splits as the direct sum of two copies of an SS which yields the $A$-summand of $1.2(\mathrm{c})$, plus a $B$-SS which yields $B_{m} \oplus B_{m}^{\prime}$ of $1.2(\mathrm{c})$. The $E_{\infty}$ term of the $A$-part of the AHSS has elements $a_{4 i+1} \otimes v_{1}^{j}, i, j \geq 0$, of order 2 , corresponding to $H_{4 i+1}(B Q) \otimes b u_{2 j}$. These have all extensions nontrivial; i.e., in an $A$-summand of $b u_{4 k+1+2 \varepsilon}(B Q), a_{4(k-j)+1} \otimes v_{1}^{2 j+\varepsilon}$ is divisible by $2^{j}$, $(\varepsilon=0,1)$. Pictorially, the SS begins as below, with a dot in position $(i, j)$ denoting $\mathbf{Z}_{2}$ in $H_{i}(B Q) \otimes b u_{j}$, and diagonal lines indicating multiplication by 2 in $b u_{*}(B Q)$ as you move up.

The $E_{\infty}$-term of the $B$-SS has elements $b_{4 i+3} \otimes v_{1}^{j}, i, j \geq 0$, of order $2^{m+1}$. It seems very difficult to determine the pattern of extensions directly. However, knowing what the answer is, i.e., 1.2(c), and that there are epimorphisms 


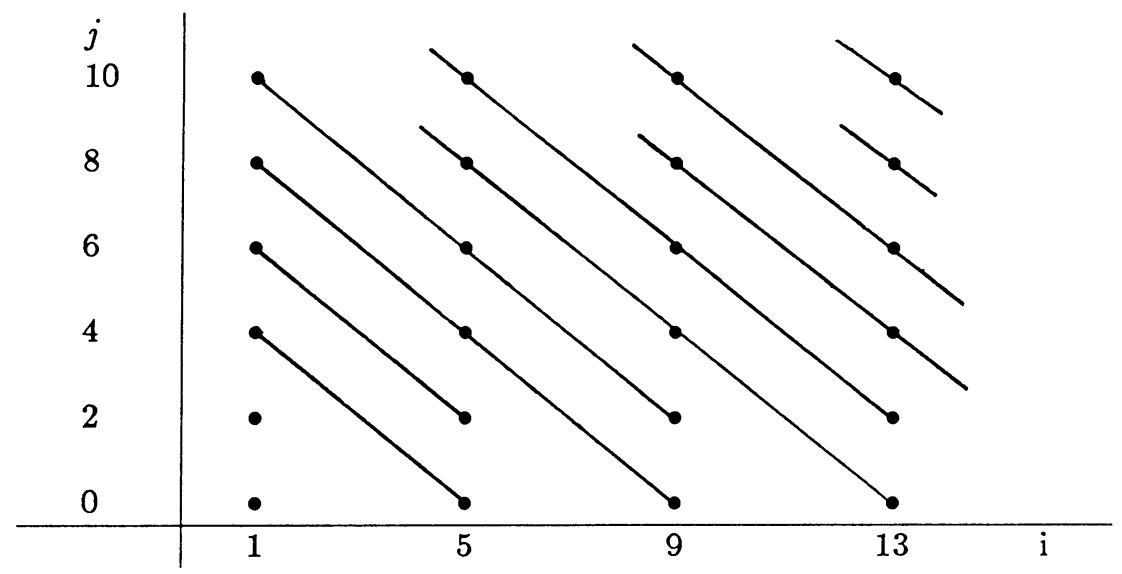

$b u_{4 n+1}(B Q) \rightarrow b u_{4 n-3}(B Q)$, which agree under 3.3 with $i^{*}: \widetilde{K}\left(S^{4 n+3} / Q\right) \rightarrow$ $\widetilde{K}\left(S^{4 n-1} / Q\right)$, which respect the SS's one can deduce the following pattern of extensions:

Let $u_{1}, \ldots, u_{n}$ denote generators of the $\mathbf{Z} / 2^{m+1}$ 's which are the $E_{\infty}$-term of the $B$-part of $\operatorname{AHSS}(B Q)$ in total degree $4 n \pm 1$. Let $\delta_{1}, \ldots, \delta_{N}$ denote generators in the splitting of the $B$-part of $b u_{4 n \pm 1}(B Q)$. (See 1.2(c), 1.3, or 3.1-3.3.) Then

$$
2^{i} \delta_{1}= \begin{cases}2^{i} u_{1}, & i \leq m, \\ 2^{m-1+\varepsilon} u_{j+2}, & i=m+2 j+1+\varepsilon, j \geq 0, \varepsilon=0,1,\end{cases}
$$

and for $2^{s-1}<j \leq 2^{s}$

$$
2^{i} \delta_{j}= \begin{cases}2^{i} u_{j}, & i<m-s, \\ 2^{m-s-1} u_{j+2^{s-1} d}, & i=m-s+d-1, d>0 .\end{cases}
$$

Of course, $u_{k}=0$ here if $k>N$.

\section{An Algebraic CONJECTURE}

The calculation of the graded abelian groups $l^{*}\left(S^{4 n+3} / Q\right)$ and $B P^{*}\left(S^{4 n+3} / Q\right)$ in $\S 3$ leads to a general algebraic question:

If $R=\mathbf{Z}\left[\left[v_{i}\right]\right]$ is a graded formal power series ring in variables $v_{i}$ of negative grading, $x$ is an indeterminate of grading 1 , and $P(x) \in R[[x]]$ is a power series of grading 1 , calculate the abelian group structure of $R[x] /\left(x^{n+1}, P(x)\right)$. We denote this abelian group by $G(n, P, R)$. See 3.1(i) and 3.8(i) for examples.

Such abelian groups also arose in our work in [BD] on cyclic groups. Here the polynomials $P(X)=\left[p^{r}\right](x)$ are obtained by iterating a series $[p](x)$, i.e., $\left[p^{r}\right](x)=[p]\left(\left[p^{r-1}\right](x)\right)$. When $p=2$,

$$
[2](x) \equiv 2 x-v_{1} x^{2} \quad \bmod \left(x^{3}\right) .
$$


A good deal of computer experimentation led the fourth author to conjecture that this information completely determines the groups $G\left(n,\left[2^{r}\right], R\right)$ for all $n$, $r$, and $R$. More generally, we have

Conjecture 4.1 (Gilkey). If $f(x)=\sum_{i \geq 1} a_{i} x^{i}$ with $a_{i} \in R_{-i+1}$ satisfies $a_{1}=2$, $a_{i} \in 2 R$ for $i<d$, and $a_{d} \notin 2 R$, and $f_{r}(x)$ is defined by $f_{1}=f$ and $f_{r}(x)=f\left(f_{r-1}(x)\right)$, then the groups $G\left(n, f_{r}, R\right)$ are completely determined by $n, d$, and $R$.

This would say that if $f=2 x+\cdots$, then the groups formed by taking quotients by iterates of $f$ are determined by the position of the first odd coefficient of $f$. The conjecture can be strengthened to give the precise groups and would give an alternative, completely algebraic, proof of Theorem 1.1 for cyclic 2-groups.

\section{A COUNTEREXAMPLE TO A CONJECTURED EXTENSION OF THEOREM 1.1}

Because the groups $G$ in 1.1 are exactly those for which

$$
\text { hom } \operatorname{dim}_{M U_{*}}\left(M U_{*}(B G)\right) \leq 1 \text {, }
$$

it was suggested in [BD] that the analog of 1.1 might be true for all spaces $X$ satisfying hom $\operatorname{dim}_{M U_{*}}\left(M U_{*}(X)\right) \leq 1$. A counterexample to this conjecture was suggested to us by D. C. Johnson.

Proposition 5.1. There is a finite complex $X$ such that hom $\operatorname{dim}_{M U_{*}}\left(M U_{*}(X)\right)$ $=1$ and $M U_{*}(X)$ is not isomorphic to $b u_{*}(X) \otimes \mathbf{Z}\left[x_{2 i}: i \geq 2\right]$.

Remark. It is still quite possible that the conjecture of [BD] might hold for all $B G$, with $G$ any finite group, i.e.,

$$
\begin{gathered}
\text { if hom } \operatorname{dim}_{B P_{*}}\left(B P_{*} B G\right) \leq n, \\
\text { then } B P_{*}(B G) \approx B P\langle n\rangle_{*}(B G) \otimes \mathbf{Z}_{(p)}\left[v_{i}: i>n\right] .
\end{gathered}
$$

If so, the question would then be: what is it about the structure of $B G$ that makes it work?

Proof. An easy Adams spectral sequence calculation shows that the 6th stable homotopy group of the real projective space $P^{4}$ is cyclic of order 2 , with nonzero element $\alpha$ a coextension of the stable map

$$
S^{6} \stackrel{\nu}{\longrightarrow} S^{3} \hookrightarrow P_{3}^{4},
$$

where $\nu$ is the stable Hopf map and $P_{3}^{4}=P^{4} / P^{2}$. Since $2 \alpha=0, \alpha$ extends to a (stable) map $S^{6} \cup_{2} e^{7} \stackrel{f}{\longrightarrow} P^{4}$. Our space $X$ is the mapping cone of $f$. Actually, a few suspensions may be required for the stable map $f$ to exist as an actual map, but we will not reflect this in our notation.

Clearly

$$
\tilde{H}_{i}(X ; \mathbf{Z}) \approx \begin{cases}\mathbf{Z}_{2}, & i=1,3,7, \\ 0, & \text { otherwise }\end{cases}
$$


Thus the AHSS converging to $M U_{*}(X)$ collapses for dimensional reasons, and so by [CS, 3.11], hom $\operatorname{dim}_{M U_{*}}\left(M U_{*}(X)\right) \leq 1$.

As $X$ is 2-primary, we shall work with $B P$ instead of $M U$. Adams spectral sequence charts for $B P_{*}(X)$ and $b u_{*}(X)$ in dimension less than 10 are pictured below.
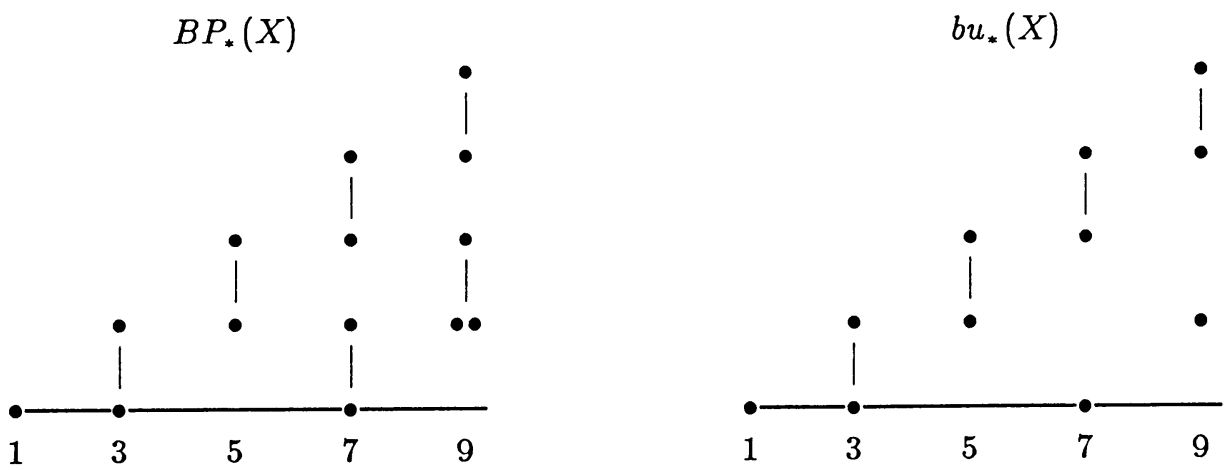

These are charts of the $E_{2}$ terms, which, by a well-known change-of-rings theorem, are isomorphic to $\operatorname{Ext}_{E}\left(H^{*} X, \mathbf{Z}_{2}\right)$ and $\operatorname{Ext}_{E_{1}}\left(H^{*} X, \mathbf{Z}_{2}\right)$, respectively where $E$ is the exterior algebra on all Milnor primitives $Q_{i}$, and $E_{1}$ is the exterior algebra on $Q_{0}$ and $Q_{1}$ (see [D]). Dots in the $i$ th column represent nonzero elements in $B P_{i}(X)$ (resp. $\left.b u_{i}(X)\right)$, and vertical lines correspond to multiplication by $h_{0}$ in Ext, which corresponds to multiplication by 2 in the generalized homology group, up to elements of higher filtration. The important fact that $h_{0}$ is nonzero on the bottom element in $B P_{7}(X)$ is seen from the relation $Q_{0} x_{7}=Q_{2} x_{1}$ in $H^{*} X$.

It is also important to our argument that there is no exotic multiplication by 2 in $b u_{7}(X)$, i.e., that $b u_{7}(X)$ is $\mathbf{Z}_{2} \oplus \mathbf{Z}_{4}$ and not $\mathbf{Z}_{8}$. This can be deduced by consideration of the complexification homomorphism $b o_{7}(X) \rightarrow b u_{7}(X)$. The only elements in $b o_{7}(X)$ are in filtrations 0 and 3 , and since the filtration 0 element maps across, there can be no extension from filtration 0 to 2 in $b u_{7}(X)$. Likewise, $B P_{7}(X) \approx \mathbf{Z}_{4} \oplus \mathbf{Z}_{4}$, since the filtration 1 and 3 classes in the possible exotic extension both come from $B P_{7}\left(P_{1}^{2}\right) \approx \mathbf{Z}_{2} \oplus \mathbf{Z}_{2}$. Thus $B P_{7}(X)$ and $b u_{7}(X) \oplus \Sigma^{6} b u_{1}(X)$ are not isomorphic.

Added in proof. An alternative discussion of the quaternionic case will appear in the Proceedings of the Northwestern Homotopy Conference, 1988.

\section{REFERENCES}

[Ad] J. F. Adams, Lectures on generalized cohomology, Lecture Notes in Math., vol. 99, SpringerVerlag, Berlin, 1969, pp. 1-138.

[At] M. F. Atiyah, K-theory, Benjamin, 1964. 
[BD] M. Bendersky and D. M. Davis, On the complex bordism of classifying spaces, Algebraic Topology, Proceedings Arcata 1986, Lecture Notes in Math., Vol. 1370, Springer-Verlag, pp. 53-56.

[BP] E. H. Brown and F. P. Peterson, A spectrum whose $\mathbf{Z}_{p}$-cohomology is the algebra of reduced pth powers, Topology 5 (1966), 149-154.

[CE] H. Cartan and S. Eilenberg, Homological algebra, Princeton Univ. Press, 1956.

[C] F. R. Cohen, Splitting certain suspensions via self-maps, Illinois J. Math. 20 (1975), 336-347.

[CF1] P. E. Conner and E. E. Floyd, Periodic maps which preserve a complex structure, Bull. Amer. Math. Soc. 70 (1964), 574-579.

[CF2] _ The relation of cobordism to $K$-theories, Lecture Notes in Math., vol. 28, SpringerVerlag, Berlin, 1966.

[CS] P. E. Conner and L. Smith, On the complex bordism of finite complexes, Publ. Math. IHES 376 (1969), 117-221.

[D] D. M. Davis, The BP-coaction for projective spaces, Canad. J. Math. 30 (1978), 45-53.

[FS] K. Fujii and M. Sugawara, The additive structure of $\widetilde{K}\left(S^{4 n+3} / Q\right)$, Hiroshima Math. J. 13 (1983), 507-521.

[G] P. Gilkey, The eta invariant and equivariant unitary bordism for spherical space form groups, Compositio Math. 65 (1988), 33-50.

[Ha] S. Hashimoto, On the connective $K$-homology groups of the classifying spaces $B Z / p^{r}$, Publ. Res. Inst. Math. Sci. 19 (1983), 765-771.

[Hu] D. Husemoller, Fiber bundles, Springer-Verlag, 1975.

[Jo] D. C. Johnson, A Stong-Hattori spectral sequence, Trans. Amer. Math. Soc. 179 (1973), 211 225.

[KS] T. Kobayashi and M. Sugawara, Note on KO-rings of lens spaces $\bmod 2^{r}$, Hiroshima Math J. 8 (1978), 85-90.

[Mes] A. Mesnaoui, Unitary bordism of classifying spaces of quaternion groups (to appear).

[MP] S. A. Mitchell and S. B. Priddy, Symmetric product spectra and splittings of classifying spaces, Amer. J. Math. 106 (1984), 219-232.

[Sw] R. G. Swan, Groups with periodic cohomology, Bull. Amer. Math. Soc. 65 (1959), 368-370.

[Wo] J. A. Wolf, Spaces of constant curvature, McGraw-Hill, New York, 1967.

Department of Mathematics, Rider College, Lawrenceville, New Jersey 08648 (Current address of Anthony Bahri)

Department of Mathematics, Hunter College, New York, New York 10021 (Current address of Martin Bendersky)

Department of Mathematics, Lehigh University, Bethlehem, Pennsylvania 18015 (Current address of D. M. Davis)

Department of Mathematics, University of Oregon, Eugene, Oregon 97403 (Current address of P. B. Gilkey) 